\title{
Impacts of Recurrent Tonsillitis on the Outcome of Powered Intracapsular Tonsillectomy in Children
}

\author{
Jeong Wook Kang, MD, Sung Hwa Dong, MD, PhD, Seon Gyu Lee, MD, and Kun Hee Lee, MD, PhD \\ Department of Otorhinolaryngology-Head and Neck Surgery, School of Medicine, Kyung Hee University, Seoul, Republic of Korea
}

Background and Objectives: To investigate the effects of recurrent tonsillitis on postoperative outcomes after powered intracapsular tonsillectomy and adenoidectomy (PITA) in children with obstructive sleep-disordered breathing (oSDB).

Materials and Methods: Children who underwent PITA in Kyung Hee University Hospital at Gangdong from 2016 to 2018 were classified into two oSDB groups according to comorbid chronic/recurrent acute tonsillitis (CHT). To evaluate the efficacy of the PITA technique, quality of life (QoL) was measured using the obstructive sleep apnea questionnaire (OSA-18) for 3 months after surgery. To evaluate the disadvantages of the PITA technique, we followed complications such as throat pain, otalgia, nausea, vomiting, and postoperative bleeding for 1 week after surgery. Finally, we compared the pre- and postoperative QoL and analyzed the incidence of postoperative complications in the oSDB with CHT (SDBwCHT) and oSDB without CHT (SDBsCHT) groups.

Results: Of the 802 enrolled patients, 135 were classified as SDBwCHT and 667 as SDBsCHT. Both groups exhibited marked improvements in QoL after PITA. The SDBwCHT group showed a change of OSA-18 score from $70.7 \pm 17.0$ to 31.2 \pm 11.4 . The SDBsCHT group showed a change of OSA-18 score from $70.0 \pm 17.1$ to $31.0 \pm 9$.9. The groups did not demonstrate statistical differences in complication rates and severity of symptoms during the first postoperative week.

Conclusion: Although comorbid CHT delayed improvement in postoperative symptoms, PITA is a useful technique to treat children with oSDB, regardless of CHT.

Keywords: Adenotonsillectomy; Tonsillectomy; Intracapsular tonsillectomy and adenoidectomy; Sleep-disordered breathing; Quality of life.

\section{INTRODUCTION}

Obstructive sleep-disordered breathing (oSDB) is a recurring condition that creates difficulty in breathing during sleep. ${ }^{1)}$ oSDB ranges from simple snoring to obstructive sleep apnea syndrome. oSDB is related to lower quality of life (QoL) subscales such as general health, physical function, behavior, somatic pain, and caregiver impact. ${ }^{2)}$ The most objective etiologic factor of oSDB in children is adenotonsillar hypertrophy. ${ }^{3)}$ From that point of view, adenotonsillectomy (T\&A) is considered the primary surgical treatment in children with oSDB. ${ }^{24)}$ At the same time, T\&A is also a favorable treatment option in

Received: April 12, 2021 Revised: May 20, 2021

Accepted: May 22, 2021

Address for correspondence: Kun Hee Lee, MD, PhD, Department of Otorhinolaryngology-Head and Neck Surgery, School of Medicine, Kyung Hee University, 892 Dongnam-ro, Gangdong-gu, Seoul 05278, Republic of Korea Tel: +82-2-440-6181, Fax:+82-2-440-7336, E-mail: nose4u@gmail.com

This is an Open Access article distributed under the terms of the Creative Commons Attribution Non-Commercial License (https://creativecommons.org/licenses/bync/4.0) which permits unrestricted non-commercial use, distribution, and reproduction in any medium, provided the original work is properly cited. children with chronic/recurrent acute tonsillitis $(\mathrm{CHT}) .{ }^{5)} \mathrm{Re}-$ moval of tonsils can prevent sore throats from recurrent acute tonsillitis. $^{5)}$

Conventional adeno-/tonsillectomy involves complete removal of the tonsil by dissecting along a plane between the tonsil capsule and the peritonsillar muscles. ${ }^{2}$ The T\&A has a long history of success and its technique is well-established. Common postoperative morbidities include bleeding and throat pain. The postoperative hemorrhagic risk is estimated to be between $2 \%$ to $7 \%{ }^{6}{ }^{6}$ Throat pain often leads to poor oral intake and dehydration. Several techniques of tonsillectomy have been introduced to reduce complications and increase patient tolerance. ${ }^{7)}$ The basic concept of the alternative techniques is intracapsular tonsil removal by preserving the tonsil capsule and a small amount of tonsil tissue. Koltai et al. ${ }^{8}$ introduced an intracapsular tonsillectomy technique using powered instrumentation that was subsequently called powered intracapsular tonsillectomy and adenoidectomy (PITA).

Since the introduction of 'intracapsular tonsillectomy and adenoidectomy' (ICTA), multiple studies have been conduct- 
ed to compare the morbidity between the conventional 'extracapsular tonsillectomy and adenoidectomy' (ECTA) and the ICTA. A recent meta-analysis showed that ICTA can be an alternative of conventional ECTA. ${ }^{9)}$ In 2020, a Cochrane review article reported that ICTA can reduce recovery time to normal activity and early postoperative complication rates. Considering PITA is a type of ICTA, postoperative morbidity may be low with the PITA technique. ${ }^{8) 10) 11)}$ Despite multiple studies discussing the benefits of PITA, it is unclear whether PITA is a favorable technique even in children with CHT. Therefore, this study aimed to analyze postoperative QoL, symptoms, and complications after PITA according to presence of CHT in children with oSDB.

\section{MATERIALS AND METHODS}

\section{Patients}

This observational cohort study included 802 children with oSDB who underwent PITA at Kyung Hee University Hospital at Gangdong between January 2016 and December 2018. Frequent events of upper airway obstruction during the night were considered oSDB history. The children were divided into two groups according to CHT history. The first CHT group consisted of 135 children with recurrent acute tonsillitis history of at least 7 in the past year; 5/year for 2 years; or 3/year for 3 years. ${ }^{2)}$ The second oSDB group consisted of 667 children with no recurrent acute tonsillitis history. Exclusion criteria were defined as follows: $<2$ years or $>16$ years of age; history of previous T\&A; genetic disorders, craniofacial abnormality; neuromuscular disorder, or mental retardation. ${ }^{2) 3}$ This study was approved by the Institutional Review Board (IRB) of Kyung Hee University Hospital at Gangdong (201604-019).

\section{Basic physical examination}

Tonsillar hypertrophy was recorded according to Brodsky's grading method. ${ }^{12)}$ Height, weight, and body mass index (BMI) were measured before surgery.

\section{Surgery and postoperative care}

All patients underwent PITA surgery under general anesthesia in the Rose position by a single senior surgeon. The surgical technique of PITA was based on Koltai et al.s method. ${ }^{8) 13)}$ The surgeon used a rubber catheter, direct mirror, and McIvor mouth gag to gain surgical view of the adenoids and tonsils. T\&A was performed using a $12^{\circ} / 40^{\circ}$ curved $4 \mathrm{~mm}$ blade microdebrider under 1,500 rpm oscillating mode (Medtronic, Minneapolis, MN, USA). Intraoperative bleeding was controlled using suction cautery or bipolar cautery. The patients received intravenous cefotetan injections with a dose of 20-30 mg/kg twice a day during admission. The patients were intraoperatively administered a $5 \mathrm{mg}$ dose of IV dexamethasone to prevent postoperative nausea and vomiting. ${ }^{2)}$ The patients were discharged with medications containing ibuprofen minus antibiotics the day after the operation. ${ }^{2)}$

\section{Postoperative quality of life}

The Korean version of the obstructive sleep apnea questionnaire was administered before and 3 months after surgery to evaluate pre- and postoperative QoL. The obstructive sleep apnea questionnaire (OSA-18) was used as a practical tool to estimate QoL impact for obstructive sleep apnea. ${ }^{14)}$ This questionnaire consisted of 18 questions divided into 5 domains of 3 to 4 questions: sleep disturbance, physical symptoms, emotional distress, daytime functions, and caregiver concerns. Every question of OSA-18 could be scored on a 1-7 point scale. The total score of OSA-18 will be assessed from 18 to 126 points.

\section{Postoperative symptoms and complications}

The Wong Baker Faces Pain Rating Scale was used as a visual analogue scale (VAS) of faces. ${ }^{15) 16)}$ Postoperative symptoms such as throat pain, otalgia, nausea, and vomiting were assessed with the VAS in the first 7 postoperative days. The medical charts of all patients were reviewed up to 1 month after surgery to record postoperative bleeding or other complications such as fever, respiratory complications, and gastrointestinal complications.

\section{Statistical analysis}

All statistical analyses were performed using R Project for Statistical Computing version 3.6.3 (http://www.r-project. org). Comparisons of demographic characteristics were analyzed with the chi-squared test, Student's t-test, and the MannWhitney U test. Comparisons of OSA-18 and symptom scale scores were analyzed with the Mann-Whitney U test. Changes of OSA-18 and symptoms according to postoperative duration were analyzed with the Wilcoxon signed-rank test. Incidence rates of postoperative complications were analyzed with the chi-squared test. Correlations between throat pain and other symptoms were analyzed with Spearman's correlation.

\section{RESULTS}

All 802 pediatric patients were classified as oSDB with CHT (SDBwCHT, $\mathrm{N}=135$ ) or oSDB without CHT (SDBsCHT, $\mathrm{N}=667$ ) subgroups. Table 1 shows the baseline characteristics of the two groups. The characteristics are similar in the two groups. The SDBwCHT children consisted of 82 males (60.7\%) and 53 females (39.3\%). The SDBsCHT children con- 
Table 1. Demographic information and duration of surgery

\begin{tabular}{lccc}
\hline \multicolumn{1}{c}{ Demographics } & $\begin{array}{c}\text { SDBwCHT } \\
(\mathrm{N}=135)\end{array}$ & $\begin{array}{c}\text { SDBsCHT } \\
(\mathrm{N}=667)\end{array}$ & $\begin{array}{c}\mathrm{p} \\
\text { value }\end{array}$ \\
\hline Sex & & & 0.555 \\
$\quad$ Male & $82(60.7)$ & $426(63.9)$ & \\
$\quad$ Female & $53(39.3)$ & $241(36.1)$ & \\
Age (years) & $5.7 \pm 2.0$ & $5.7 \pm 2.1$ & 0.890 \\
Height $(\mathrm{cm})$ & $116.3 \pm 12.9$ & $116.2 \pm 14.4$ & 0.953 \\
Weight $(\mathrm{kg})$ & $23.4 \pm 8.0$ & $23.5 \pm 9.9$ & 0.899 \\
BMI (kg/m $\left.{ }^{2}\right)$ & $16.8 \pm 2.4$ & $16.7 \pm 2.8$ & 0.688 \\
Right tonsil size & & & 0.661 \\
$1+$ & $0(0.0)$ & $5(0.7)$ & \\
$2+$ & $6(4.4)$ & $21(3.1)$ & \\
$3+$ & $15(11.1)$ & $72(10.8)$ & \\
$4+$ & $114(84.4)$ & $569(85.3)$ & \\
Left tonsil size & & & 0.607 \\
$1+$ & $0(0.0)$ & $2(0.3)$ & \\
$2+$ & $5(3.7)$ & $14(2.1)$ & \\
$3+$ & $18(13.3)$ & $81(12.1)$ & \\
$4+$ & $112(83.0)$ & $570(85.5)$ & \\
Duration of surgery (min) & $20.0 \pm 7.0$ & $20.2 \pm 7.0$ & 0.847
\end{tabular}

Data presented as mean \pm standard deviation or number (\%). SDBwCHT, obstuctive sleep-disordered breathing with chronic tonsillitis; SDBsCHT, obstuctive sleep-disordered breathing without chronic tonsillitis; BMI, body mass index

sisted of 426 males (63.9\%) and 241 females (36.1\%). The mean age of the two groups was 5.7 years. The mean height and weight of the two groups were approximately $116 \mathrm{~cm}$ and 23 $\mathrm{kg}$, respectively. The mean BMI was approximately $16 \mathrm{~kg} / \mathrm{m}^{2}$. The mean duration of surgery was about 20 minutes. The two groups exhibited no statistical differences in sex ratio, age, height, weight, BMI, tonsil size, and duration of surgery.

Table 2 represents the comparison of symptoms and OSA18 at the same point in time between the two groups. The two groups did not exhibit statistical differences in OSA-18 during the pre-/postoperative period. There were no statistical differences in postoperative throat pain, otalgia, nausea, and vomiting during the first week after surgery.

Table 3 shows QoL improvements by OSA-18. The SDBwCHT children showed a change of OSA-18 score from 70.7 \pm 17.0 to $31.2 \pm 11.4$. The SDBsCHT children showed a decrease of OSA-18 score from 70.0 \pm 17.1 to $31.0 \pm 9.9$. Both groups exhibited statistical improvement of postoperative OSA-18 3 months after surgery.

Table 4 shows the VAS scores of the symptoms at a specific time after surgery. Throat pain was statistically improved for the first 24 hours after surgery in the SDBsCHT group but not SDBwCHT group. The SDBwCHT group showed a significant 1-week delay for relief of pain. Otalgia was paradoxically increased 1-week after surgery in SDBsCHT but not in
Table 2. Comparison of OSA-18 and symptom scale scores before and after surgery

\begin{tabular}{lccc}
\hline \multicolumn{1}{c}{ Variable } & $\begin{array}{c}\text { SDBwCHT } \\
(\mathrm{N}=135)\end{array}$ & $\begin{array}{c}\text { SDBsCHT } \\
(\mathrm{N}=667)\end{array}$ & $\begin{array}{c}\mathrm{p} \\
\text { value }\end{array}$ \\
\hline OSA-18 & & & \\
$\quad$ Before surgery & $70.7 \pm 17.0$ & $70.0 \pm 17.1$ & 0.692 \\
3 months after surgery & $31.2 \pm 11.4$ & $31.0 \pm 9.9$ & 0.879 \\
12 hours after surgery & & & \\
$\quad$ Throat pain & $4.4 \pm 2.6$ & $4.4 \pm 2.8$ & 0.790 \\
Otalgia & $0.6 \pm 1.5$ & $0.5 \pm 1.3$ & 0.132 \\
Nausea & $0.8 \pm 1.9$ & $1.2 \pm 2.3$ & 0.406 \\
Vomiting & $0.2 \pm 0.8$ & $0.2 \pm 0.7$ & 0.172 \\
24 hours after surgery & & & \\
Throat pain & $4.2 \pm 2.8$ & $4.0 \pm 2.7$ & 0.902 \\
Otalgia & $0.6 \pm 1.7$ & $0.4 \pm 1.3$ & 0.543 \\
Nausea & $0.7 \pm 1.9$ & $0.6 \pm 1.6$ & 0.758 \\
Vomiting & $0.1 \pm 0.5$ & $0.1 \pm 0.5$ & 0.423 \\
1 week after surgery & & & \\
Throat pain & $1.8 \pm 1.9$ & $1.7 \pm 1.9$ & 0.623 \\
Otalgia & $0.6 \pm 1.6$ & $0.6 \pm 1.4$ & 0.563 \\
Nausea & $0.2 \pm 0.9$ & $0.3 \pm 0.9$ & 0.357 \\
Vomiting & $0.1 \pm 0.4$ & $0.1 \pm 0.6$ & 0.299 \\
\hline
\end{tabular}

Data presented as mean \pm standard deviation. SDBwCHT, obstuctive sleep-disordered breathing with chronic tonsillitis; SDBsCHT, obstuctive sleep-disordered breathing without chronic tonsillitis; OSA-18, obstructive sleep apnea-18

Table 3. Changes in OSA-18 scores before surgery and at 3 months after surgery

\begin{tabular}{cccc}
\hline \multicolumn{1}{c}{ OSA-18 } & $\begin{array}{c}\text { Before } \\
\text { surgery }\end{array}$ & $\begin{array}{c}3 \text { months } \\
\text { after surgery }\end{array}$ & $\begin{array}{c}\mathrm{p} \\
\text { value }\end{array}$ \\
\hline SDBwCHT $(\mathrm{N}=135)$ & $70.7 \pm 17.0$ & $31.2 \pm 11.4$ & $<0.001^{*}$ \\
SDBsCHT $(\mathrm{N}=667)$ & $70.0 \pm 17.1$ & $31.0 \pm 9.9$ & $<0.001^{*}$ \\
\hline
\end{tabular}

Data presented as mean \pm standard deviation. *statistical significance. SDBwCHT, obstuctive sleep-disordered breathing with chronic tonsillitis; SDBsCHT, obstuctive sleep-disordered breathing without chronic tonsillitis; OSA-18, obstructive sleep apnea-18

SDBwCHT. The SDBsCHT children showed early improvements within 24 hours in nausea and vomiting. The SDBw$\mathrm{CHT}$ children exhibited delayed improvement in nausea and no improvement in vomiting.

Table 5 shows complication events before and 24 hours postoperatively. Bleeding events were slightly increased in the SDBwCHT group but were not statistically different compared with the SDBsCHT group. Other complications were abundant in the SDBsCHT group but again were not statistically different compared with the SDBwCHT group.

Fig. 1 shows the correlations between throat pain and other symptoms such as otalgia and nausea. Children who underwent PITA exhibited statistically significant correlations 
Table 4. Changes in symptom scores at 12 hours, 24 hours, and 1 week after surgery

\begin{tabular}{|c|c|c|c|c|c|c|}
\hline Symptom scores & $\begin{array}{c}12 \text { hours } \\
\text { after surgery }\end{array}$ & $\begin{array}{c}24 \text { hours } \\
\text { after surgery }\end{array}$ & $\begin{array}{c}1 \text { week } \\
\text { after surgery }\end{array}$ & p value ${ }^{\dagger}$ & $\mathrm{p}$ value $\ddagger$ & $\mathrm{p}$ value ${ }^{\S}$ \\
\hline \multicolumn{7}{|l|}{ Throat pain } \\
\hline SDBwCHT $(\mathrm{N}=135)$ & $4.4 \pm 2.6$ & $4.2 \pm 2.8$ & $1.8 \pm 1.9$ & 0.357 & $<0.001^{*}$ & $<0.001^{*}$ \\
\hline SDBsCHT $(\mathrm{N}=667)$ & $4.4 \pm 2.8$ & $4.0 \pm 2.7$ & $1.7 \pm 1.9$ & $<0.001^{*}$ & $<0.001^{*}$ & $<0.001^{*}$ \\
\hline \multicolumn{7}{|l|}{ Otalgia } \\
\hline SDBwCHT $(\mathrm{N}=135)$ & $0.6 \pm 1.5$ & $0.6 \pm 1.7$ & $0.6 \pm 1.6$ & $>0.999$ & $>0.999$ & 0.733 \\
\hline SDBsCHT $(\mathrm{N}=667)$ & $0.5 \pm 1.3$ & $0.4 \pm 1.3$ & $0.6 \pm 1.4$ & 0.083 & $0.031^{*}$ & $0.006^{*}$ \\
\hline \multicolumn{7}{|l|}{ Nausea } \\
\hline SDBwCHT $(\mathrm{N}=135)$ & $0.8 \pm 1.9$ & $0.7 \pm 1.9$ & $0.2 \pm 0.9$ & 0.322 & $<0.001^{*}$ & $0.016^{*}$ \\
\hline SDBsCHT $(\mathrm{N}=667)$ & $1.2 \pm 2.3$ & $0.6 \pm 1.6$ & $0.3 \pm 0.9$ & $<0.001^{*}$ & $<0.001^{*}$ & $<0.001^{*}$ \\
\hline \multicolumn{7}{|l|}{ Vomiting } \\
\hline SDBwCHT $(\mathrm{N}=135)$ & $0.2 \pm 0.8$ & $0.1 \pm 0.5$ & $0.1 \pm 0.4$ & 0.896 & 0.492 & 0.676 \\
\hline SDBsCHT $(\mathrm{N}=667)$ & $0.2 \pm 0.7$ & $0.1 \pm 0.5$ & $0.1 \pm 0.6$ & $<0.001^{*}$ & $0.017^{*}$ & 0.451 \\
\hline
\end{tabular}

Data presented as mean \pm standard deviation. ${ }^{*}$ statistical significance; ${ }^{\dagger} 12$ hours vs. 24 hours; ${ }^{\ddagger} 12$ hours vs. 1 week; $\$ 24$ hours vs. 1 week. SDBwCHT, obstuctive sleep-disordered breathing with chronic tonsillitis; SDBsCHT, obstuctive sleep-disordered breathing without chronic tonsillitis

Table 5. Frequency of complications by the post-operative time

\begin{tabular}{lcrr}
\hline \multicolumn{1}{c}{ Complications } & $\begin{array}{c}\text { SDBwCHT } \\
(\mathrm{N}=135)\end{array}$ & $\begin{array}{c}\text { SDBsCHT } \\
(\mathrm{N}=667)\end{array}$ & $\begin{array}{r}\mathrm{p} \\
\text { value }\end{array}$ \\
\hline Within 24 hours & $2(1.5)$ & $11(1.6)$ & $>0.999$ \\
Bleeding & $2(1.5)$ & $2(0.3)$ & 0.134 \\
Other adverse events & $0(0)$ & $9(1.3)$ & 0.370 \\
After 24 hours & $5(3.7)$ & $28(4.2)$ & 0.979 \\
Bleeding & $5(3.7)$ & $14(2.0)$ & 0.419 \\
Other adverse events & $0(0)$ & $14(2.1)$ & 0.144 \\
\hline
\end{tabular}

Data are presented as $\mathrm{n}(\%)$. Other adverse events include fever, respiratory complication, and gastrointestinal complication. SDBwCHT, obstuctive sleep-disordered breathing with chronic tonsillitis; SDBsCHT, obstuctive sleep-disordered breathing without chronic tonsillitis

between throat pain and otalgia at all times (Fig. 1A-C). They also showed a correlation between throat pain and nausea (Fig. 1D-F).

\section{DISCUSSION}

The concept of PITA surgery involves removal of as much tonsillar tissue as possible while preserving the tonsillar capsule. The tonsillar capsule and a thin layer of remaining tonsillar tissue can act as a 'biological dressing' to protect the peritonsillar muscle layers from damage and reduce inflammatory reactions. ${ }^{17)}$ The biological dressing also can reduce thermal damage from electrocauterization devices. Sorin et al. ${ }^{7}$ reported that tonsillar regrowth with snoring was the most common complication $(3.2 \%)$ in children who underwent PITA (N=278). Acute streptococcal-positive tonsillitis appears to exacerbate tonsillar regrowth. ${ }^{7}$ A recent study using conventional tonsillectomy reported that postoperative bleeding rate was higher in the $\mathrm{CHT}$ group compared to the SDB group. ${ }^{18)}$ Although some studies have shown the benefits of extracapsular tonsillectomy, multiple studies have failed to demonstrate any statistical differences in disease-specific QoL improvements between conventional tonsillectomy and intracapsular tonsillectomy in children with oSDB. ${ }^{3)}$ In addition, it is unclear what pros and cons can be expected when extracapsular tonsillectomy is performed in patients with CHT.

\section{QoL}

The OSA-18 questionnaire has been widely used to assess post-adenotonsillectomy QoL in children with oSDB.$^{19-21)}$ The OSA-18 survey is a validated instrument that is correlated with tonsil size, adenoid size, and respiratory distress index in children with oSDB. ${ }^{14)}$ The present authors could not determine any statistical differences of the pre-/postoperative QoL of PITA surgery between the SDBwCHT group and the SDBsCHT group from the Korean version OSA-18 questionnaire (Table 2). According to the tonsillectomy guideline of the American Academy of Otolaryngology-Head and Neck Surgery (AAO-HNS), frequent tonsillitis can be an independent indication of adenotonsillectomy. ${ }^{2)}$ Children with only CHT and not SDB are clinically uncommon. Moreover, most patients did not have well-written medical records to prove pharyngitis episodes. For these reasons, we selected children with SDBwCHT and not children with CHT. In our study, diseasespecific QoL improved not only in the SDBsCHT group, but also in the SDBwCHT group (Table 3 and Supplementary Fig. 1). The lack of statistical differences of pre-/postoperative QoL and QoL improvements in both groups indicates that 


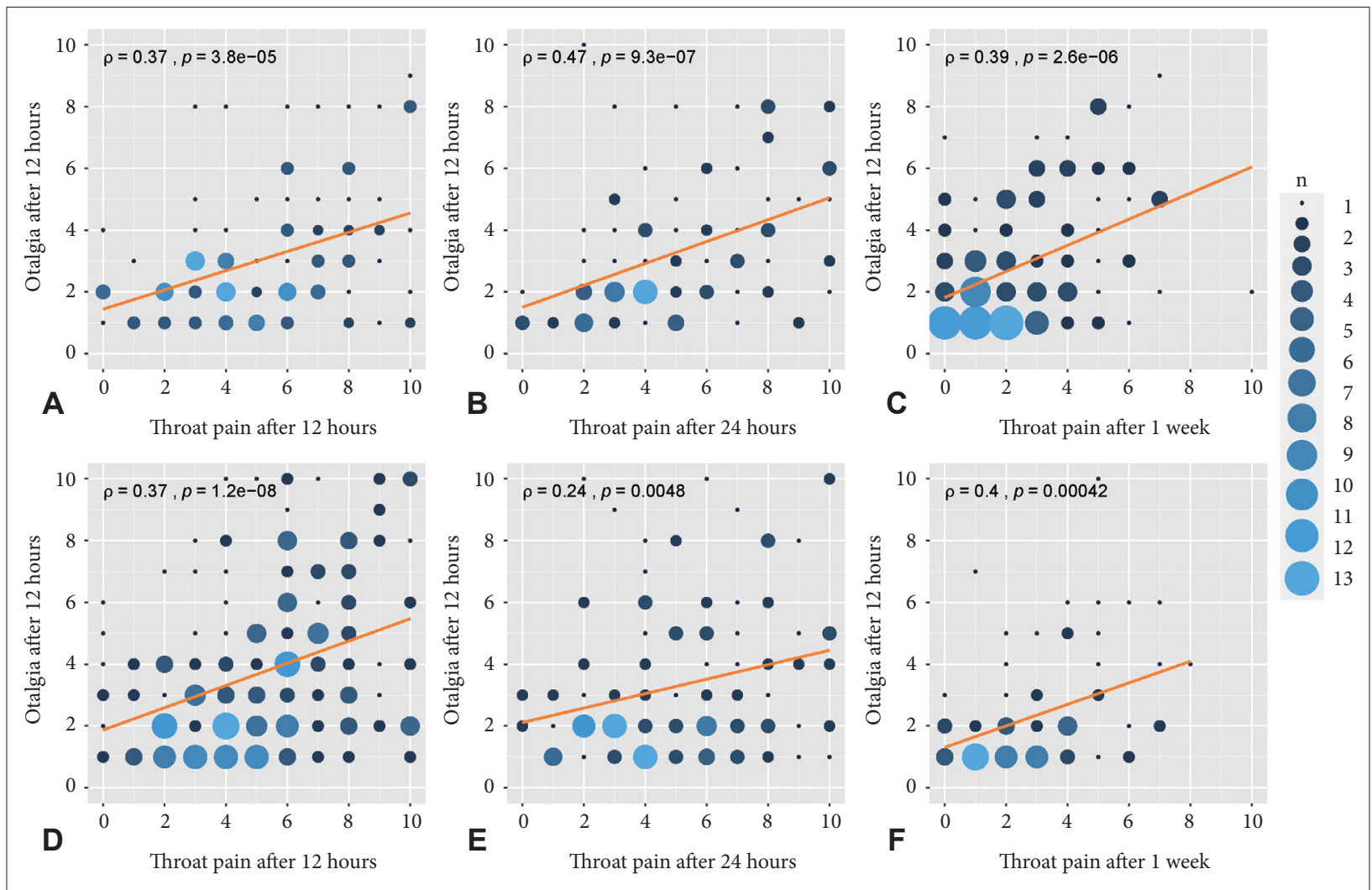

Fig. 1. Correlation between throat pain and other symptoms. Patients without otalgia or nausea were excluded. Correlation between throat pain and otalgia $(A, B, C)$. Correlation between throat pain and nausea (D, E, F). $\rho$ : Spearman's correlation coefficient.

PITA surgery is an effective technique for patients with oSDB regardless of history of recurrent tonsillitis (Tables 2 and 3).

\section{Symptoms}

This study followed up postoperative symptoms such as otalgia, nausea, and vomiting during 1 week after PITA (Tables 2 and 4). Throat pain is a main cause of morbidity after tonsillectomy, resulting in poor oral intake, dehydration, sleep disturbance, or other behavioral changes. ${ }^{2)}$ Therefore, AAOHNS guidelines recommend perioperative pain counseling and postoperative pain control. ${ }^{2)}$ Crandall et al. ${ }^{22)}$ reported a gradual decrease of the numeric $0-10$ pain intensity scale during the 3 days after the tonsillectomy procedure. In that study, throat pain was recorded on a 4-5 pain intensity scale and was observed to not be relieved during the first two postoperative days. ${ }^{22)}$ In our study, throat pain exhibited a slight change up to the first 24 hours after surgery and was greatly relieved 1 week after surgery (Tables 2 and 4). Although children with oSDBsCHT showed slightly faster relief of pain within 24 hours in this study, it was not a clinically meaningful outcome because throat pain exhibited no differences between oSDBsCHT and oSDBwCHT (Tables 2 and 4).

Sensory innervation of the ear includes cranial nerves $\mathrm{V}$,
VII, IX, and X and spinal nerves C2 and C3. ${ }^{23)}$ Tonsillectomyrelated referred otalgia can arise from cranial nerve IX innervating the tonsillar bed. ${ }^{24)}$ Referred otalgia is a common symptom during the same period of postoperative throat pain. ${ }^{25)}$ In our study, continuity of referred otalgia pain was questionable. Many patients with no otalgia in the first 12 hours complained of otalgia 1 week after surgery (Supplementary Fig. 2). The incidence rate of referred otalgia was increased in children with throat pain (Supplementary Table 1). Unlike throat pain that lasted up to 1 week after surgery, otalgia incidentally occurred as a sore throat condition. Once a patient experienced referred otalgia, the severity of otalgia was correlated to the severity of throat pain (Fig. 1). Although the incidence rates of nausea and vomiting were lower compared to the incidence rate of otalgia, such symptoms exhibited similar occurrence patterns observed in otalgia (Fig. 1, Supplementary Table 1 and Supplementary Fig. 2).

\section{Complications}

Post-tonsillectomy hemorrhage is a serious complication with risk of mortality. ${ }^{26)}$ Postoperative bleeding is classified as either primary bleeding within 24 hours after surgery or secondary bleeding 24 hours postoperatively. ${ }^{2)}$ Primary bleeding 
has been reported to have an incidence rate of $0.2 \%$ to $2.2 \%$, while secondary bleeding has been from $0.1 \%$ to $3 \%{ }^{27)}$ Postoperative bleeding frequently occurs between 5 and 10 days after surgery. ${ }^{6)}$ Two reviews reported less postoperative bleeding in intracapsular tonsillectomy. ${ }^{9 / 28)}$ Perkins et al. ${ }^{29)}$ showed that patients with OSA have a lower postoperative bleeding rate than chronic tonsillitis patients. However, we were unable to reproduce the results (Table 5). We believe that the incidence of bleeding was too low. Therefore, if the number of subjects increases, the difference may be statistically significant (Table 5).

\section{Limitations}

This study has several limitations. First, the follow-up period of this study is relatively short considering the duration of postoperative pain. We followed up symptoms for just 1 week and OSA-18 for 3 months. According to the guideline of AAOHNS, pain after tonsillectomy on average lasts 7-10 days but can last as long as 2 weeks. ${ }^{2)}$ A systematic review revealed that short- and long-term scores of OSA-18 exhibited no significant difference. ${ }^{30)}$ Second, when we categorized patients into oSDBsCHT and oSDBwCHT, we used OSA-18 rather than overnight polysomnography (PSG). Not all hospitals are able to use PSG for diagnosis of oSDB in children due to limitations of medical facilities and manpower. In addition, our study aimed to evaluate QoL after surgery and not the numerical values of PSG. Third, because this study did not include children who underwent extracapsular tonsillectomy, it was impossible to compare intracapsular and extracapsular tonsillectomies. We must presuppose the results of other studies showing to demonstrate the advantages of the PITA technique. ${ }^{3)}$ Finally, our results have the limitations of a single-center study. To exclude selection bias, multicentered prospective randomized trials are necessary.

\section{Conclusion}

Although relief of postoperative throat pain could be a little faster in children with oSDBsCHT, there was no statistical difference in post-PITA QoL, symptoms, and complications between oSDBwCHT and oSDBsCHT groups. Therefore, PITA is an applicable surgical technique for children with oSDB regardless of CHT.

\section{Supplementary Materials}

The online-only Data Supplement is available with this article at https://doi.org/10.18787/jr.2021.00355.

\section{Conflicts of Interest}

The authors have no potential conflicts of interest to disclose.

\section{Author Contributions}

Conceptualization: Kun Hee Lee. Data curation: Seon Gyu Lee. Formal analysis: Jeong Wook Kang, Sung Hwa Dong, Seon Gyu Lee. Methodology: Sung Hwa Dong. Project administration: Kun Hee Lee. Software: Jeong Wook Kang. Supervision: Kun Hee Lee. Writing_original draft: Jeong Wook Kang. Writing_-review \& editing: Kun Hee Lee.

\section{ORCID iD}

Kun Hee Lee https://orcid.org/0000-0002-5380-3726

Funding Statement

None

\section{REFERENCES}

1) Venekamp RP, Hearne BJ, Chandrasekharan D, Blackshaw H, Lim J, Schilder AG. Tonsillectomy or adenotonsillectomy versus non-surgical management for obstructive sleep-disordered breathing in children. Cochrane Database Syst Rev 2015;10:CD011165.

2) Baugh RF, Archer SM, Mitchell RB, Rosenfeld RM, Amin R, Burns JJ, et al. Clinical practice guideline: tonsillectomy in children. Otolaryngol Head Neck Surg 2011;144(1 Suppl):S1-30.

3) Blackshaw H, Springford LR, Zhang LY, Wang B, Venekamp RP, Schilder AG. Tonsillectomy versus tonsillotomy for obstructive sleep-disordered breathing in children. Cochrane Database Syst Rev 2020;4(4): CD011365.

4) Marcus CL, Brooks LJ, Draper KA, Gozal D, Halbower AC, Jones J, et al. Diagnosis and management of childhood obstructive sleep apnea syndrome. Pediatrics 2012;130(3):e714-55.

5) Burton MJ, Glasziou PP, Chong LY, Venekamp RP. Tonsillectomy or adenotonsillectomy versus non-surgical treatment for chronic/recurrent acute tonsillitis. Cochrane Database Syst Rev 2014;2014(11): CD001802.

6) Krishna P, Lee D. Post-tonsillectomy bleeding: a meta-analysis. Laryngoscope 2001;111(8):1358-61.

7) Sorin A, Bent JP, April MM, Ward RF. Complications of microdebrider-assisted powered intracapsular tonsillectomy and adenoidectomy. Laryngoscope 2004;114(2):297-300.

8) Koltai PJ, Solares CA, Mascha EJ, Xu M. Intracapsular partial tonsillectomy for tonsillar hypertrophy in children. Laryngoscope 2002; 112(S100):17-9.

9) Kim JS, Kwon SH, Lee EJ, Yoon YJ. Can intracapsular tonsillectomy be an alternative to classical tonsillectomy? A meta-analysis. Otolaryngol Head Neck Surg 2017;157(2):178-89.

10) Lee HS, Yoon HY, Jin HJ, Hwang SH. The safety and efficacy of powered intracapsular tonsillectomy in children: a meta-analysis. Laryngoscope 2018;128(3):732-44.

11) Kordeluk S, Goldbart A, Novack L, Kaplan DM, El-Saied S, Alwalidi $\mathrm{M}$, et al. Randomized study comparing inflammatory response after tonsillectomy versus tonsillotomy. Eur Arch Otorhinolaryngol 2016; 273(11):3993-4001.

12) Ng SK, Lee DL, Li AM, Wing YK, Tong MC. Reproducibility of clinical grading of tonsillar size. Arch Otolaryngol Head Neck Surg 2010; 136(2):159-62.

13) Koltai PJ, Chan J, Younes A. Power-assisted adenoidectomy: total and partial resection. Laryngoscope 2002;112(S100):29-31.

14) Franco RA Jr, Rosenfeld RM, Rao M. First place--resident clinical science award 1999. Quality of life for children with obstructive sleep apnea. Otolaryngol Head Neck Surg 2000;123(1 Pt 1):9-16.

15) Garra G, Singer AJ, Domingo A, Thode HC Jr. The Wong-Baker pain FACES scale measures pain, not fear. Pediatr Emerg Care 2013;29(1): 17-20.

16) Tomlinson D, von Baeyer CL, Stinson JN, Sung L. A systematic review 
of faces scales for the self-report of pain intensity in children. Pediatrics 2010;126(5):e1168-98.

17) Derkay CS, Darrow DH, Welch C, Sinacori JT. Post-tonsillectomy morbidity and quality of life in pediatric patients with obstructive tonsils and adenoid: microdebrider vs electrocautery. Otolaryngol Head Neck Surg 2006;134(1):114-20.

18) Rodríguez-Catalán J, Fernández-Cantalejo Padial J, Rodríguez Rodríguez P, González Galán F, Del-Río Camacho G. Postoperative complications after adenotonsillectomy in two paediatric groups: obstructive sleep apnoea syndrome and recurrent tonsillitis. Acta Otorrinolaringol Esp (Engl Ed) 2020;71(1):32-9.

19) Borgström A, Nerfeldt $P$, Friberg D. Adenotonsillotomy versus adenotonsillectomy in pediatric obstructive sleep apnea: an RCT. Pediatrics 2017;139(4):e20163314.

20) Ericsson E, Lundeborg I, Hultcrantz E. Child behavior and quality of life before and after tonsillotomy versus tonsillectomy. Int J Pediatr Otorhinolaryngol 2009;73(9):1254-62.

21) Spruyt K, Gozal D. Pediatric sleep questionnaires as diagnostic or epidemiological tools: a review of currently available instruments. Sleep Med Rev 2011;15(1):19-32.

22) Crandall M, Lammers C, Senders C, Braun JV, Savedra M. Children's pre-operative tonsillectomy pain education: clinical outcomes. Int J Pediatr Otorhinolaryngol 2008;72(10):1523-33.

23) Anwar K, Khan S, Shahabi I, Niazi ZB. The frequency of involvement of head \& neck sites in referred otalgia - An experience at a tertiary care hospital. Pak J Med Sci 2019;35(4):1138-42.

24) Charlett SD, Coatesworth AP. Referred otalgia: a structured approach to diagnosis and treatment. Int J Clin Pract 2007;61(6):1015-21.

25) Eriksson M, Nilsson U, Bramhagen AC, Idvall E, Ericsson E. Self-reported postoperative recovery in children after tonsillectomy compared to tonsillotomy. Int J Pediatr Otorhinolaryngol 2017;96:47-54.

26) Ingram DG, Friedman NR. Toward adenotonsillectomy in children: a review for the general pediatrician. JAMA Pediatr 2015;169(12):115561.

27) Windfuhr JP, Chen YS, Remmert S. Hemorrhage following tonsillectomy and adenoidectomy in 15,218 patients. Otolaryngol Head Neck Surg 2005;132(2):281-6.

28) Wang H, Fu Y, Feng Y, Guan J, Yin S. Tonsillectomy versus tonsillotomy for sleep-disordered breathing in children: a meta analysis. PloS One 2015;10(3):e0121500.

29) Perkins JN, Liang C, Gao D, Shultz L, Friedman NR. Risk of post-tonsillectomy hemorrhage by clinical diagnosis. Laryngoscope 2012;122(10): 2311-5.

30) Todd CA, Bareiss AK, McCoul ED, Rodriguez KH. Adenotonsillectomy for obstructive sleep apnea and quality of life: systematic review and meta-analysis. Otolaryngol Head Neck Surg 2017;157(5): 767-73. 